\title{
Article \\ Fractal features of Fermi Blazar Light Curves
}

\author{
Y. Liu ${ }^{1,2, *}$, W. Cai ${ }^{1,2}$ and J. H. Fan ${ }^{1,2}$ \\ 1 Center for Astrophysics, Guangzhou University, Guangzhou 510006, China \\ 2 Astronomy Science and Technology Research Laboratory of Department of Education of Guangdong \\ Province, Guangzhou 510006, China \\ * Correspondence: pinux@gzhu.edu.cn
}

\begin{abstract}
In this work, we'll present the power spectra density, auto-correlation function, dentrended fluctuation analysis and multifractal spectrum are performed to the daily light curves of Blazars monitored by Fermi LAT. Some signs of power law are found.
\end{abstract}

Keywords: galaxies:blazar; light curve; simulation

\section{Introduction}

A blazar is a very compact active galactic nuclei (AGN) associated with a supermassive black hole. Blazars emit a relativistic jet that is pointing in the direction of us, which accounts for the rapid variability. Blazars show extreme observation phenomena: such as luminous emissions, rapid and high amplitude variability, high and variable polarization, superluminal features etc. There are two subclasses of Blazars, one is BL Lacertae objects (BLs), another is flat spectrum radio quasars (FSRQs). A physical classification of blazars can be made by using their peak frequency $v_{p}$ in the spectral energy distribution (SEDs) [1,2]. Abdo et al. extended the definition to all non-thermal dominated AGNs using new acronyms as low synchrotron peaked blazars (LSP, $\log v_{p}<14 \mathrm{~Hz}$ ), intermediate synchrotron peaked blazars (ISP, $\log v_{p}=14 \sim 15 \mathrm{~Hz}$ ), and high synchrotron peaked blazars (HSP, $\log v_{p}>15 \mathrm{~Hz}$ ) [3]. Recently, Fan et al. obtained the SEDs for a sample of 1425 Fermi blazars and gave new results, LSP with $\log v_{p}<14.0 \mathrm{~Hz}$; ISP with $\log v_{p}=14.0 \sim 15.3 \mathrm{~Hz}$; HSP with $\log v_{p}>15.3 \mathrm{~Hz}[4]$.

Variability is one of the main observational feature of blazars, showing at the whole electromagnetic wave-bands. Timescales of the variations across multiple orders of magnitude, from less than one hour up to sveral decades [5], in other words, the variations is present at almost every time scale.

\section{Fermi Data}

All our data were downloaded from the website of Fermi science support Center. The Fermi Large Area Telescope (Fermi-LAT), is an imaging, wide field-of view, $\gamma$-ray telescope. It is usually operated in a sky surveying mode, mapping the full sky every 3 hours. This provides a regular monitoring of all the gamma-bright objects in the sky, most of them are blazars. A Fermi LAT daily light curve of 3C 273 is plotted in Figure 1, shows very complex variations. 


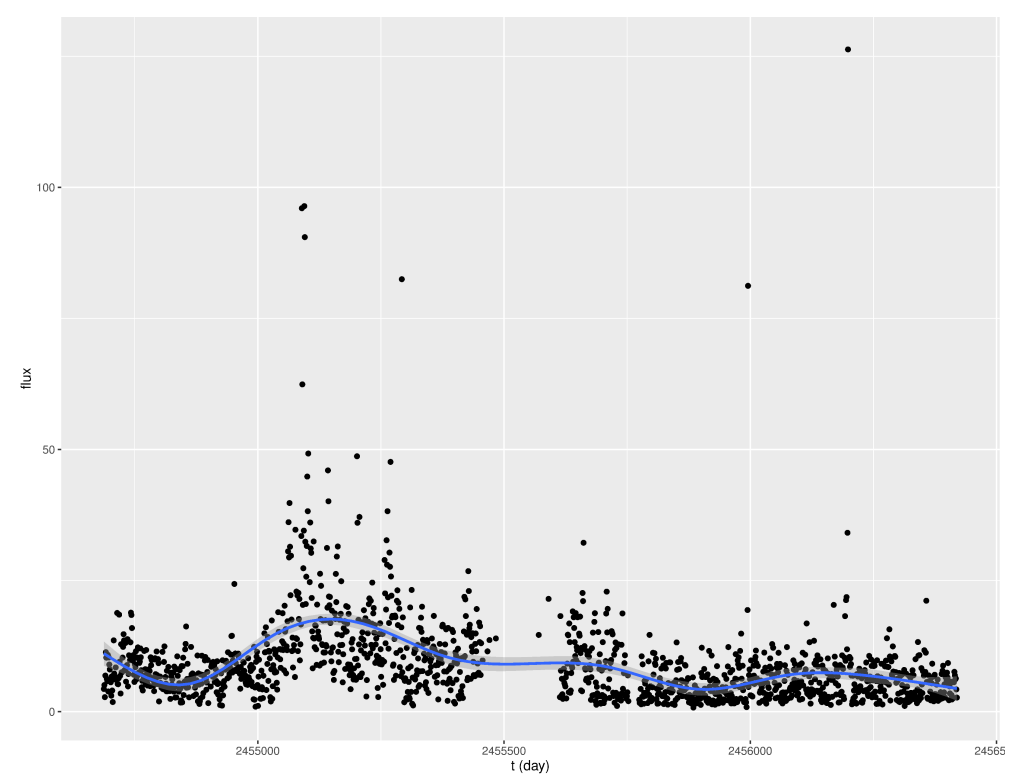

Figure 1. Fermi LAT daily light curve of 3C 273. A smooth line is plotted.

\section{Analysis methods}

In this work, the Power spectral density (PSD) method is used to obtain 1st power-law index between the PSD intensity $p s d$ and frequency $f$, the Auto-correlation Function (ACF) method is used to obtain 2nd power-law index between the ACF intensity acf and time lag $\tau$, the Dentrended Fluctuation Analysis (DFA) is used to obtain 3rd power-law index between the DFA intensity $d f a$ and timescale $s$, the Multifractral spectra (MFS) is also used. As an example, the PSD, ACF, DFA and MFS of 3C 273 are plotted in Figure 2.

Many attempts of PSD for unevenly sampled light curve have been made to investigate the periodicity [7]. A improved technigue is the Date-Compensated Discrete Fourier Transform [8], a least-square regression on sine, cosine and constant function. The DCDFT is a powerful method for astronomy light curve.

ACF is the similarity between light curves as a function of the time lag between them. It is a useful tool for finding repeating patterns. The ACF and PSD are traditional methods for capturing long-range power correlation.

DFA is a method for determining the statistical self-affinity of a light curve, it's widly used in stochastic processes, chaos theory and time series analysis. It is useful for analysing time series that appear to be long-memory processes (diverging correlation time, e.g. power-law decaying autocorrelation function) or $1 / f$ noise.

Based on DFA, multi-fractal detrended fluctuation analysis (MF-DFA) has been introduced and successfully applied in many fields. The MFS can be obtrained by using MF-DFA. MFS is used to check the scaling features of Fermi daily light curve. 

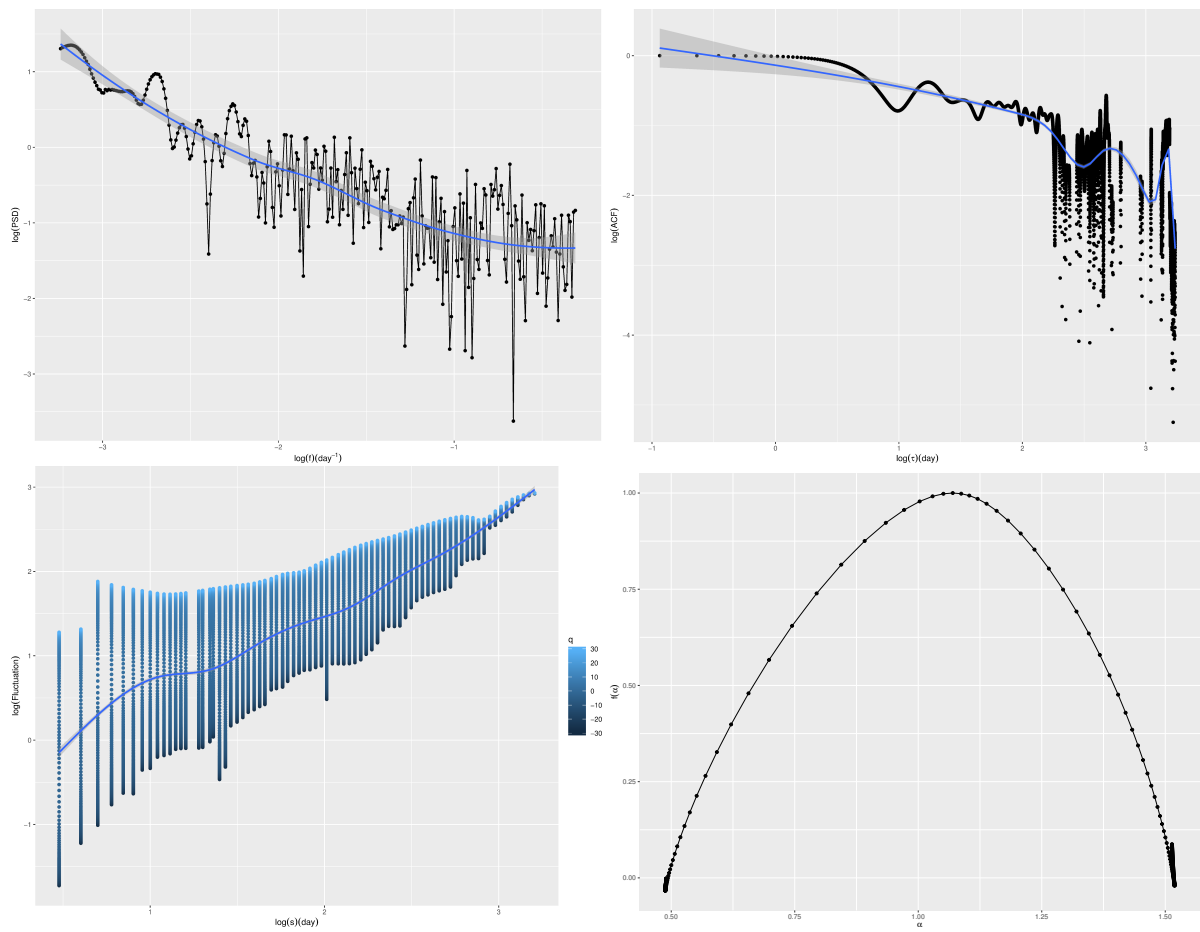

Figure 2. (a) Power spetral density of Fermi light curve of $3 C 273$, psd $\propto f^{-0.93}$; (b) Auto-corelation Function of Fermi light curve of 3C 273, acf $\propto \tau^{-0.70}$; (c) Dentrended Fluctuation Analysis of Fermi light curve of 3 C 273, $d f a \propto s^{0.94}$; (d) Multifractral spectra of Fermi light curve of 3C 273.

\section{Discussions and Conclusions}

PSD, ACF and DFA power law index have been obtained. Based on power law index of PSD, the average value is 0.76 , therefore, most Fermi-LAT light curves should be similar to pink noise (or $1 / f$ noise), not red noise. From the results of ACF, the long-range dependency feature should be found. Integrated PSD and DFA, it can be seen that the Fermi-LAT daily light curves are more possibly belong to fractal Gaussian process. MFS shows that the light curves have self-similar feature, it is a multifractal time series.

Acknowledgments: The work is partially supported by the National Natural Science Foundation of 122 China (NSFC U1531245, U1431112, U11203007, 11403006, 10633010, 11173009), the Innovation Foundation of 123 Guangzhou University (IFGZ), Guangdong Province Universities and Colleges Pearl River Scholar Funded 124 Scheme(GDUPS)(2009), Yangcheng Scholar Funded Scheme(10A027S), and support for Astrophysics Key 125 Subjects of Guangdong Province and Guangzhou City. The Abastumani team acknowledges financial support 126 of the project FR/639/6-320/12 by the Shota Rustaveli National Science Foundation under contract 31/76

Author Contributions: YLiu is responsible for the writing and the motivation of the paper; CWei is for some analysis of light curves; JHFan is responsible for some discussions.

Conflicts of Interest: The authors declare no conflict of interest.

\section{References}

1. Padovani, P.; Giommi, P. The connection between x-ray- and radio-selected BL Lacertae objects. ApJ, 1995, $444,567-581$.

2. Urry, C.M.; Padovani, P. Unified Schemes for Radio-Loud Active Galactic Nuclei. PASP, 1995, 107, 803-845.

3. Abdo, A. A.; Ackermann, M.; Agudo, I.; et al. The Spectral Energy Distribution of Fermi Bright Blazars. ApJ, 2010, 716, 30-70.

4. Fan, J. H.; Yang, J. H.; Liu, Y.; Luo, G.Y.; Lin, C.; Yuan, Y.H.; Xiao, H.B.; Zhou, A. Y.; Hua, T.X.; Pei, Z.Y. The Spectral Energy Distributions of Fermi Blazars. ApJS, 2016, (in press).

5. Fan, J. H. Optical Variability of Blazars. ChJAA (RAA), 2005, 5, 213-223. 
6. Ackermann, M., et al., The Second Catalog of Active Galactic Nuclei Detected by the Fermi Large Area Telescope, 2011, ApJ., 743, 171

7. Ferraz-Mello, S. Estimation of Periods from Unequally Spaced Observations. AJ, 1981, 86, 619-624.

8. Foster, G. The cleanest Fourier spectrum. AJ, 1995, 109, 1889-1902.

(C) 2016 by the authors; licensee Preprints, Basel, Switzerland. This article is an open access article distributed under the terms and conditions of the Creative Commons Attribution (CC-BY) license (http://creativecommons.org/licenses/by/4.0/). 